\title{
Radiographic findings in 240 patients with COVID-19 pneumonia: time-dependence after the onset of symptoms
}

\author{
Sergio Giuseppe Vancheri ${ }^{1,2} \cdot$ Giovanni Savietto $^{1,2} \cdot$ Francesco Ballati $^{1,2} \cdot$ Alessia Maggi $^{1,2} \cdot$ Costanza Canino $^{3}$. \\ Chandra Bortolotto $^{1}$ - Adele Valentini ${ }^{1}$ - Roberto Dore ${ }^{4}$. Giulia Maria Stella ${ }^{5}$ - Angelo Guido Corsico ${ }^{6}$. \\ Giorgio Antonio lotti ${ }^{7,8} \cdot$ Francesco Mojoli $^{7,8}$ - Stefano Perlini ${ }^{9,10}$ - Raffaele Bruno ${ }^{11}$ - Lorenzo Preda ${ }^{1,2}$
}

Received: 1 April 2020 / Revised: 30 April 2020 / Accepted: 14 May 2020 / Published online: 30 May 2020

(C) European Society of Radiology 2020

\begin{abstract}
Objective To analyze the most frequent radiographic features of COVID-19 pneumonia and assess the effectiveness of chest Xray $(\mathrm{CXR})$ in detecting pulmonary alterations.

Materials and methods CXR of 240 symptomatic patients ( $70 \%$ male, mean age $65 \pm 16$ years), with SARS-CoV-2 infection confirmed by RT-PCR, was retrospectively evaluated. Patients were clustered in four groups based on the number of days between symptom onset and CXR: group A (0-2 days), 49 patients; group B (3-5), 75 patients; group C (6-9), 85 patients; and group D (>9), 31 patients. Alteration's type (reticular/ground-glass opacity (GGO)/consolidation) and distribution (bilateral/ unilateral, upper/middle/lower fields, peripheral/central) were noted. Statistical significance was tested using chi-square test. Results Among 240 patients who underwent CXR, 180 (75\%) showed alterations (group A, 63.3\%; group B, 72\%; group C, $81.2 \%$; group D, $83.9 \%)$. GGO was observed in $124 / 180$ patients $(68.8 \%)$, reticular alteration in $113 / 180(62.7 \%)$, and consolidation in 71/180 (39.4\%). Consolidation was significantly less frequent $(p<0.01)$. Distribution among groups was as follows: reticular alteration (group A, 70.9\%; group B, 72.2\%; group C, 57.9\%; group D, 46.1\%), GGO (group A, 67.7\%; group B, $62.9 \%$; group C, $71 \%$; group D, $76.9 \%$ ), and consolidation (group A, 35.5\%; group B, 31.4\%; group C, 47.8\%; group D, 38.5\%). Alterations were bilateral in $73.3 \%$. Upper, middle, and lower fields were involved in $36.7 \%, 79.4 \%$, and $87.8 \%$, respectively. Lesions were peripheral in $49.4 \%$, central in $11.1 \%$, or both in $39.4 \%$. Upper fields and central zones were significantly less involved $(p<0.01)$.

Conclusions The most frequent lesions in COVID-19 patients were GGO (intermediate/late phase) and reticular alteration (early phase) while consolidation gradually increased over time. The most frequent distribution was bilateral, peripheral, and with middle/lower predominance. Overall rate of negative CXR was $25 \%$, which progressively decreased over time.
\end{abstract}

Giovanni Savietto

giovannisavietto@gmail.com

1 Department of Radiology, I.R.C.C.S. Policlinico San Matteo Foundation, Viale Camillo Golgi, 19, Pavia 27100, Italy

2 Unit of Radiology, Department of Clinical, Surgical, Diagnostic, and Pediatric Sciences, University of Pavia, Viale Camillo Golgi, 19, 27100 Pavia, Italy

3 Medical Oncology Unit, Fondazione I.R.C.C.S. Policlinico San Matteo, Pavia, Italy

4 Radiology Unit, Isituti Clinici Città di Pavia, Pavia, Italy

5 Unit of Respiratory Diseases, Department of Internal Medicine and Therapeutics, I.R.C.C.S. Policlinico San Matteo Foundation, Viale Camillo Golgi, 19, Pavia 27100, Italy
6 Unit of Respiratory Diseases, Department of Internal Medicine and Therapeutics, University of Pavia and I.R.C.C.S. Policlinico San Matteo Foundation, Viale Camillo Golgi, 19, Pavia 27100, Italy

7 Unit Anesthesiology, Department of Clinical, Surgical, Diagnostic and Pediatric Sciences, University of Pavia, Viale Camillo Golgi, 19, Pavia 27100, Italy

8 Anesthesia and Intensive Care Unit 1, Department of Intensive Medicine, I.R.C.C.S. Policlinico San Matteo Foundation, Viale Camillo Golgi, 19, Pavia 27100, Italy

9 Department of Internal Medicine and Therapeutics, University of Pavia, Viale Camillo Golgi, 19, Pavia 27100, Italy

10 Emergency Department, I.R.C.C.S. Policlinico San Matteo Foundation, Viale Camillo Golgi, 19, Pavia 27100, Italy

11 Department of Infectious and Tropical Diseases, University of Pavia and I.R.C.C.S. Policlinico San Matteo Foundation, Viale Camillo Golgi, 19, Pavia 27100, Italy 


\section{Key Points}

- The predominant lung changes were GGO and reticular alteration, while consolidation was less frequent.

- The typical distribution pattern was bilateral, peripheral, or both peripheral and central and involved predominantly the lower and middle fields.

- Chest radiography showed lung abnormalities in $75 \%$ of patients with confirmed SARS-CoV-2 infection, range varied from 63.3 to $83.9 \%$, respectively, at 0-2 days and $>9$ days from the onset of symptoms.

Keywords Severe acute respiratory syndrome coronavirus $2 \cdot$ Radiography $\cdot$ COVID-19 $\cdot$ Pneumonia $\cdot$ Thorax

\author{
Abbreviations \\ CXR Chest X-ray \\ GGO Ground-glass opacity \\ RT-PCR Reverse transcription polymerase chain reaction
}

\section{Introduction}

Since December 2019, when the first cluster of cases of coronavirus disease 2019 (COVID-19) was reported in Wuhan, Hubei Province, China, the widespread transmission of coronavirus type 2 (SARS-CoV-2) has reached pandemic proportions [1]. The manifestations of SARS-CoV-2 infection in humans range from mild respiratory symptoms to severe acute respiratory syndrome $[2,3]$. According to the WHO, several molecular assays based on reverse transcription polymerase chain reaction (RT-PCR) for the individuation of SARSCoV-2 genes are recommended for the confirmation of COVID-19 [4]. The role of imaging in the diagnosis of COVID-19 is still under debate. Several early radiological studies emphasized the role of radiological imaging in the early detection and management of COVID-19. These studies analyzed and described the chest computed tomography (CT) findings at the presentation and at different times throughout the disease course $[5,6]$. During the subsequent outbreak in the Western world, chest X-ray (CXR), together with arterial blood gas analysis and clinical presentation, in patients positive to RT-PCR, was recommended as a useful and easily available tool to support the initial diagnosis and for the subsequent management of COVID-19 patients [7, 8]. Nonetheless, data specifically addressing CXR findings in COVID-19 are still limited [9-11]. Also, the role of lung ultrasound is probably valuable but still unclear [12].

In order to create reasonable imaging workflows, it is important to evaluate the diagnostic potential of CXR and that radiologists become familiar with the presentation pattern of COVID-19 in CXR. Our study aimed to evaluate the percentage of abnormal chest radiographs at different time intervals from the onset of symptoms and to identify the type and distribution of radiographic alterations and their frequency at different times throughout the disease course of COVID-19 pneumonia.

\section{Materials and methods}

\section{Patients}

The inclusion criteria of this retrospective study were as follows: (1) patients with confirmed SARS-CoV-2 infection, in which the SARS-CoV-2 infection was confirmed by real-time RT-PCR on nasopharyngeal swab or bronchoalveolar lavage (BAL) specimens, according to international guidelines [4]; (2) knowledge of the precise date of onset of symptoms considered linked to the onset of viral pneumonia such as fever $\left(>37.5^{\circ} \mathrm{C}\right)$, cough, and dyspnea [2] and asymptomatic patients were excluded from the study; and (3) patients who underwent CXR examination.

All patients underwent CXR and RT-PCR on the day of admission in the emergency department. Two investigators collected the clinical data regarding the time of onset of symptoms from the digital archive of the emergency department of all the patients suspected for COVID-19 admitted at Fondazione IRCCS Policlinico San Matteo between February 21 and March 8, 2020.

The same two investigators subsequently noted the results and the date of RT-PCR of each patient. IRB approval was obtained for this study.

Informed consent for processing personal data for research purposes was obtained from each patient.

\section{Image acquisition and evaluation}

All the patients underwent a baseline digital anteroposterior bedside chest radiography at full inspiration using a portable radiography unit (FDR Go, Fujifilm Corporation). X-ray examinations were reviewed independently by two experienced thoracic radiologists (R.D. and L.P., with more than 30 and 15 years of experience, respectively). Results were compared, and when disagreement was found, final decisions were determined by consensus.

The findings considered in the evaluation of CXR were the presence or absence and the type of pulmonary alterations, and their distribution. 
Fig. 1 The three main alterations on chest radiography (upper line) and the corresponding findings on chest CT (lower line). Left:

diffuse reticular alteration (arrows). The corresponding CT shows diffuse increased lung attenuation and interlobular septal thickening (arrows). Middle: peripheral ground-glass opacities (arrows). Right: extensive consolidations (arrows). The corresponding CT shows predominant consolidative alterations (arrows)
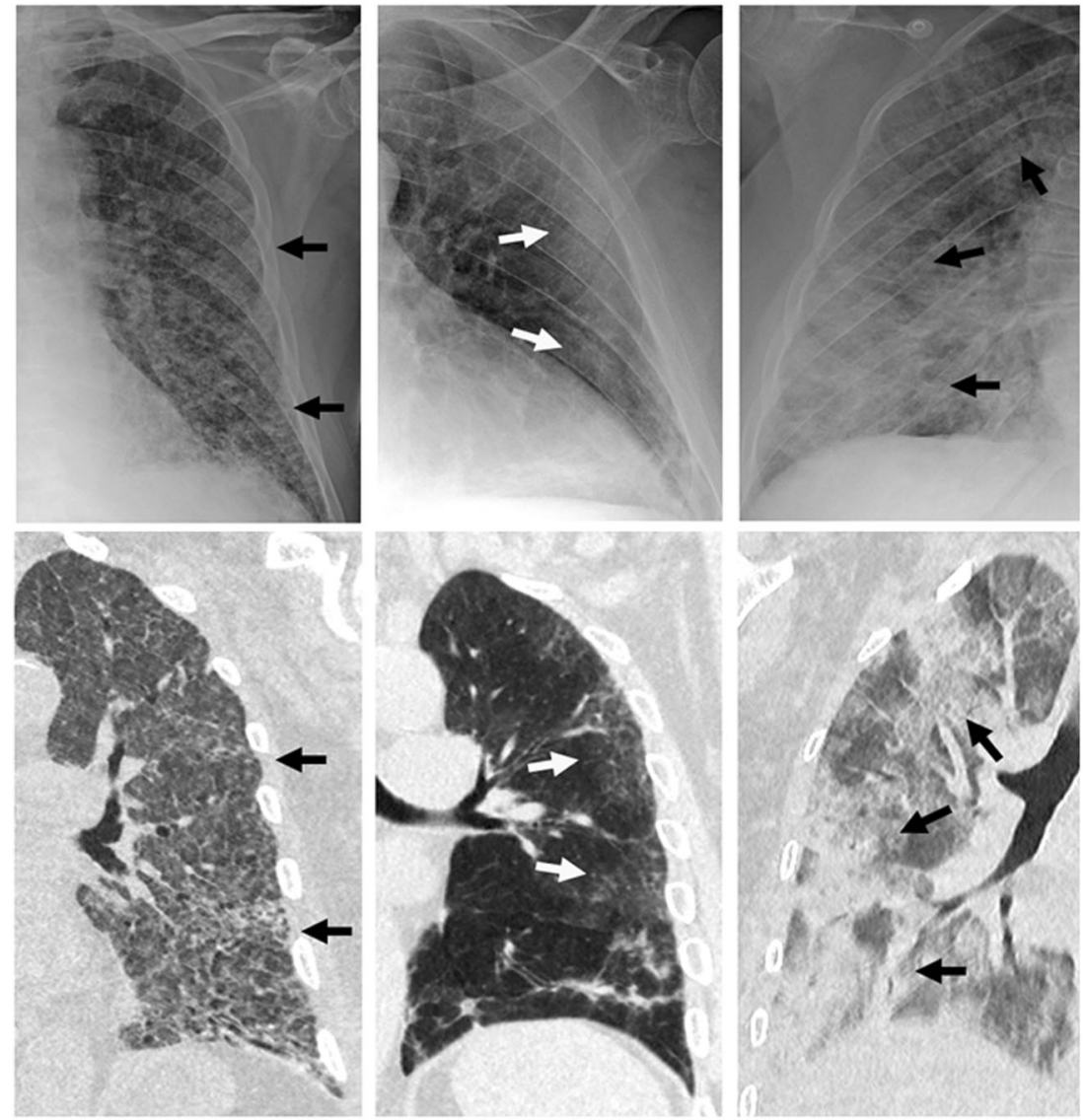

CXR alterations (Fig. 1) were defined according to the Fleischner Society's nomenclature, available in the Glossary of Terms for Thoracic Imaging [13]:

- Reticular alteration, as a collection of innumerable small linear opacities that, by summation, produce an appearance of a net;

- Consolidation, as a homogeneous increase in pulmonary parenchymal attenuation that obscures the margins of the vessels and airway walls;

- Ground-glass opacity (GGO), as an area of hazy, increased lung opacity, usually extensive, within which margins of pulmonary vessels may be indistinct.

The distribution of alterations was classified as unilateral or bilateral. Within each hemithorax, the craniocaudal distribution of the lesions was evaluated on the basis of the involvement of the upper, middle, and lower fields. The middle field was defined as the lung area delimited by (included between) two horizontal lines at the level of the superior and inferior hilar horns, respectively; the superior field was defined as the lung area included between the horizontal line at the level of the upper hilar horn and the apical pleura; the lower field was defined as the lung area included between the horizontal line at the level of the inferior hilar horn and the diaphragm.

The horizontal distribution of the lesions was evaluated on the basis of the involvement of the peripheral zone only, the central zone only, or both. Central zones were defined as the central area within $2 \mathrm{~cm}$ from the lobar bronchial structures as far as visible; peripheral zones were defined as the remaining lung area between the central zones and the pleura [14].

\section{Subgrouping of patients}

The patients were clustered into four groups based on the number of days between the onset of symptoms and the chest radiography: group A (patients with chest radiographs acquired 0-2 days from the onset of symptoms), group B (patients with chest radiographs acquired 3-5 days from the onset of symptoms), group C (patients with chest radiographs acquired 6-9 days from the onset of symptoms), and group D (patients with chest radiographs acquired over 9 days from the onset of symptoms).

\section{Statistical analysis}

Statistical analyses were performed using MedCalc for Windows, version 15.0 (MedCalc software). Continuous 
variables were expressed as mean $\pm \mathrm{SD}$ values. The frequency of the radiographic findings was expressed as the number of occurrences and percentage in every single cluster. Frequencies in the different groups were compared using the chi-square test; $p$ values $<0.05$ were considered significant.

\section{Results}

\section{Patient characteristics}

Among 305 patients initially considered, 60 patients were excluded because the onset of symptoms was uncertain and 5 patients were excluded because they were asymptomatic. A total of 240 patients was considered; among them, $169(70 \%)$ were men and $71(30 \%)$ were women. The average age was $65 \pm 16$ years (range 16-97 years). Numerosity and demographics of the subgroups are summarized in Table 1.

\section{Chest radiography evaluation}

Among the total of 240 patients, $180(75 \%)$ had at least one alteration in one lung field. In 60/240 patients (25\%), CXR was normal without any lesion. CXR showed at least one alteration in at least one lung field in $31 / 49$ patients $(63 \%)$ in group $\mathrm{A}$, in $54 / 74$ patients $(72 \%)$ in group $\mathrm{B}$, in $69 / 85$ patients $(81 \%)$ in group $\mathrm{C}$, and in $26 / 31$ patients $(83 \%)$ in group $\mathrm{D}$. The negative rate of chest radiographs was $18 / 49$ patients $(36.7 \%)$ in group A, 21/75 (28\%) in group B, 16/ $85(18.8 \%)$ in group $\mathrm{C}$, and $5 / 31(16.1 \%)$ in group D. Alterations were bilateral in $132 / 180$ patients $(73.3 \%)$ and unilateral in 48/180 (26.6\%) (24 in the left lung and 24 in the right lung). GGO, alone or in combination with other alterations, was present in $124 / 180$ patients $(68.8 \%)$; reticular alteration, alone or in combination with other findings, was present in 113/180 patients (62.7\%); consolidation, alone or in combination with other findings, was present in $71 / 180$ patients $(39.4 \%)$. GGO and reticular alteration were significantly more frequent than consolidation ( $p<0.01$ in both cases) Table 2 . A significantly higher frequency of involvement of the lower fields compared to the middle fields and of the lower and middle fields compared to the upper fields was observed $(p<0.01$ in all cases) Table 3. The exclusive involvement of the central zones was significantly less frequent than the exclusive involvement of the peripheral zones and than the involvement of both peripheral and central zones $(p<0.01$ in both cases) Table 3.

Pleural effusion was observed in $12 / 180$ patients $(6.6 \%)$, unilateral in all cases ( 7 on the right side and 5 on the left side).

\section{Chest radiography evaluation of subgroups}

Bilateral alterations were present in $25 / 31$ patients $(80.6 \%)$ in group $\mathrm{A}$, in $36 / 54$ patients $(66.6 \%)$ in group $\mathrm{B}$, in $51 / 69$ patients $(73.9 \%)$ in group $C$, and in $20 / 26$ patients $(76.9 \%)$ in group D; when alterations were unilateral, no significant difference was observed between the left and right lungs.

GGO, alone or in combination with other alterations, was present in 21/31 patients (67.7\%) in group A, in 34/54 patients (62.9\%) in group $\mathrm{B}$, in $49 / 69$ patients $(71 \%)$ in group $\mathrm{C}$, and in 20/26 patients $(76.9 \%)$ in group D.

Reticular alteration, alone or in combination with other alterations, was present in 22/31 patients (70.9\%) in group $\mathrm{A}$, in $39 / 54$ patients $(72.2 \%)$ in group B, in $40 / 69$ patients (57.9\%) in group C, and in $12 / 26$ patients (46.1\%) in group D.

Consolidation, alone or in combination with other alterations, was present in $11 / 31$ patients $(35.5 \%)$ in group $\mathrm{A}$, in $17 / 54$ patients $(31.4 \%$ ) in group $\mathrm{B}$, in $33 / 69$ patients $(47.8 \%)$ in group C, and in $10 / 26$ patients $(38.5 \%)$ in group D (Figs. 2a-d and 3).

The specific frequency of the different alterations and their associations are summarized in Table 2 .

The upper fields were involved in $12 / 31$ patients $(38.7 \%)$ in group $\mathrm{A}$, in $19 / 54(35.2 \%)$ in group $\mathrm{B}$, in $25 / 69(36.2 \%)$ in group $\mathrm{C}$, and in $10 / 26(38.5 \%)$ in group $\mathrm{D}$; the middle fields were involved in $27 / 31$ patients $(87.1 \%$ ) in group $\mathrm{A}$, in $41 / 54$ (75.9\%) in group $\mathrm{B}$, in $56 / 69(81.1 \%)$ in group $\mathrm{C}$, and in $19 / 26$ $(73.1 \%)$ in group D; the lower fields were involved in $28 / 31$ patients $(90.3 \%)$ in group $\mathrm{A}$, in $46 / 54(85.2 \%)$ in group $\mathrm{B}$, in $60 / 69(86.9 \%)$ in group C, and in 24/26 (92.3\%) in group D.

The exclusive involvement of the peripheral zones was observed in $19 / 31$ patients $(61.3 \%$ ) in group A, in 26/54 $(48.1 \%)$ in group B, in 28/69 (40.6\%) in group C, and in 16/ $26(61.5 \%)$ in group D; the involvement of both peripheral

Table 1 Patient characteristics

\begin{tabular}{|c|c|c|c|c|c|}
\hline & All patients $(n=240)$ & Group A $(n=49)$ & Group B $(n=75)$ & Group C $(n=85)$ & Group D $(n=31)$ \\
\hline Mean age \pm SD & $65 \pm 16.3$ & $66 \pm 16.8$ & $63 \pm 17.2$ & $63 \pm 14.5$ & $67 \pm 9.2$ \\
\hline \multicolumn{6}{|l|}{ Sex, $n(\%)$} \\
\hline Men & $169(70)$ & $32(65)$ & $52(69)$ & $62(73)$ & $23(74)$ \\
\hline Women & $71(30)$ & $17(35)$ & $23(31)$ & $23(27)$ & $8(26)$ \\
\hline
\end{tabular}


Table 2 Radiographic alterations

\begin{tabular}{|c|c|c|c|c|c|}
\hline & All patients $(n=240)$ & Group A $(n=49)$ & Group B $(n=75)$ & Group C $(n=85)$ & Group $\mathrm{D}(n=31)$ \\
\hline \multirow[t]{2}{*}{ Negative chest radiography, $n(\%)$} & $60(25)$ & $18(36.7)$ & $21(28)$ & $16(18.8)$ & $5(16.1)$ \\
\hline & All patients $(n=180)$ & Group A $(n=31)$ & Group B $(n=54)$ & Group C $(n=69)$ & Group D $(n=26)$ \\
\hline \multicolumn{6}{|l|}{ Positive chest radiographs, $n(\%)$} \\
\hline Bilateral alterations & $132(73.3)$ & $25(80.6)$ & $36(66.6)$ & $51(73.9)$ & $20(76.9)$ \\
\hline Pleural effusion & $12(6.6)$ & $2(6.4)$ & $4(7.4)$ & $4(5.8)$ & $2(7.7)$ \\
\hline Reticular alteration (total) & $113(62.7)$ & $22(70.9)$ & $39(72.2)$ & $40(57.9)$ & $12(46.1)$ \\
\hline Ground-glass opacity (total) & $124(68.8)$ & $21(67.7)$ & $34(62.9)$ & $49(71)$ & $20(76.9)$ \\
\hline Consolidation (total) & $71(39.4)$ & $11(35.5)$ & $17(31.4)$ & $33(47.8)$ & $10(38.5)$ \\
\hline Reticular alteration alone & $27(15)$ & $5(16.1)$ & $13(24.1)$ & $7(10.1)$ & $2(7.7)$ \\
\hline Ground-glass opacity alone & $38(21.1)$ & $5(16.1)$ & $11(20.3)$ & $14(20.3)$ & $8(30.8)$ \\
\hline Consolidation alone & $8(4.4)$ & $1(3.2)$ & 0 & $5(7.2)$ & $2(7.6)$ \\
\hline
\end{tabular}

and central zones was observed in $11 / 31$ patients $(35.5 \%)$ in group $\mathrm{A}$, in $21 / 54(38.9 \%)$ in group $\mathrm{B}$, in $31 / 69$ (44.9\%) in group $\mathrm{C}$, and in $8 / 26$ (30.8\%) in group $\mathrm{D}$; the exclusive involvement of the central zones was observed in $1 / 31$ patients (3\%) in group $\mathrm{A}$, in $7 / 54(12.9 \%)$ in group $\mathrm{B}$, in $10 / 69$ (14.5\%) in group C, and in 2/26 (7\%) in group D (Fig. 4).

\section{Discussion}

In early clinical experience, chest $\mathrm{CT}$ has been considered the most reliable imaging modality to support the diagnosis in suspected cases of COVID-19 [15]. Chest CT showed high sensitivity in detecting GGO, which is considered a typical finding in COVID-19 pneumonia and, in some cases, may be the only alteration present in the early phases of the disease
$[3,16]$. CXR was not recommended as a first-line imaging examination due to its low sensitivity in detecting alterations $[5,15]$.

Conversely, recent statements of several influential radiological societies $[7,8,17-22]$ recommend that CT should not be used as a first-line tool to support the diagnosis of COVID19 , and encourage the use of CXR in combination with RTPCR test.

Our study is one of the first to address this issue and systematically describe the CXR findings at the admission in COVID-19 pneumonia in a vast population $[9,10]$. Salehi et al [11] analyzed several studies on initial and follow-up imaging in COVID-19, performed a meta-analysis on 919 patients who underwent chest $\mathrm{CT}$, and reported general considerations on CXR. Wong et al [9] reviewed 255 CXR in 64 patients, describing the time course of the radiographic

Table 3 Significant differences in the frequency of the alterations and their distribution

\begin{tabular}{lcc}
\hline & $p$ value & $95 \%$ CI \\
\hline Alterations (alone or in combination) & $<0.01^{*}$ & $13.0-32.9$ \\
Reticular $113 / 180(62.7 \%)>$ consolidation $71 / 180(39.4 \%)$ & $<0.01^{*}$ & $19.3-38.8$ \\
GGO $124 / 180(68.8 \%)>$ consolidation $71 / 180(39.4 \%)$ & 0.2 & -3.6 to 15.8 \\
GGO $124 / 180(68.8 \%)>$ reticular $113 / 180(62.7 \%)$ & \\
Lung field involvement & $<0.01^{*}$ \\
Field involvement & $<0.01^{*}$ \\
$\quad$ Middle $143 / 180(79.4 \%)>$ upper $66 / 180(36.7 \%)$ & 0.03 & $33.0-51.2$ \\
Lower $158 / 180(87.8 \%)>$ upper $66 / 180(36.7 \%)$ & $41.9-58.9$ \\
Lower $158 / 180(87.8 \%)>$ middle $143 / 180(79.4 \%)$ & $0.7-16.0$ \\
Zone involvement & $<0.01^{*}$ \\
Peripheral $89 / 180(49.4 \%)>$ central $20 / 180(11.1 \%)$ & $<0.01^{*}$ & $29.3-46.5$ \\
Both $71 / 180(39.4 \%)>$ central $20 / 180(11.1 \%)$ & 0.06 & $10.2-26.4$ \\
Peripheral $89 / 180(49.4 \%)>$ both $71 / 180(39.4 \%)$ & -0.3 to 20 \\
\hline
\end{tabular}

$G G O$ ground-glass opacity

$* p$ values $<0.05$ were considered significant 

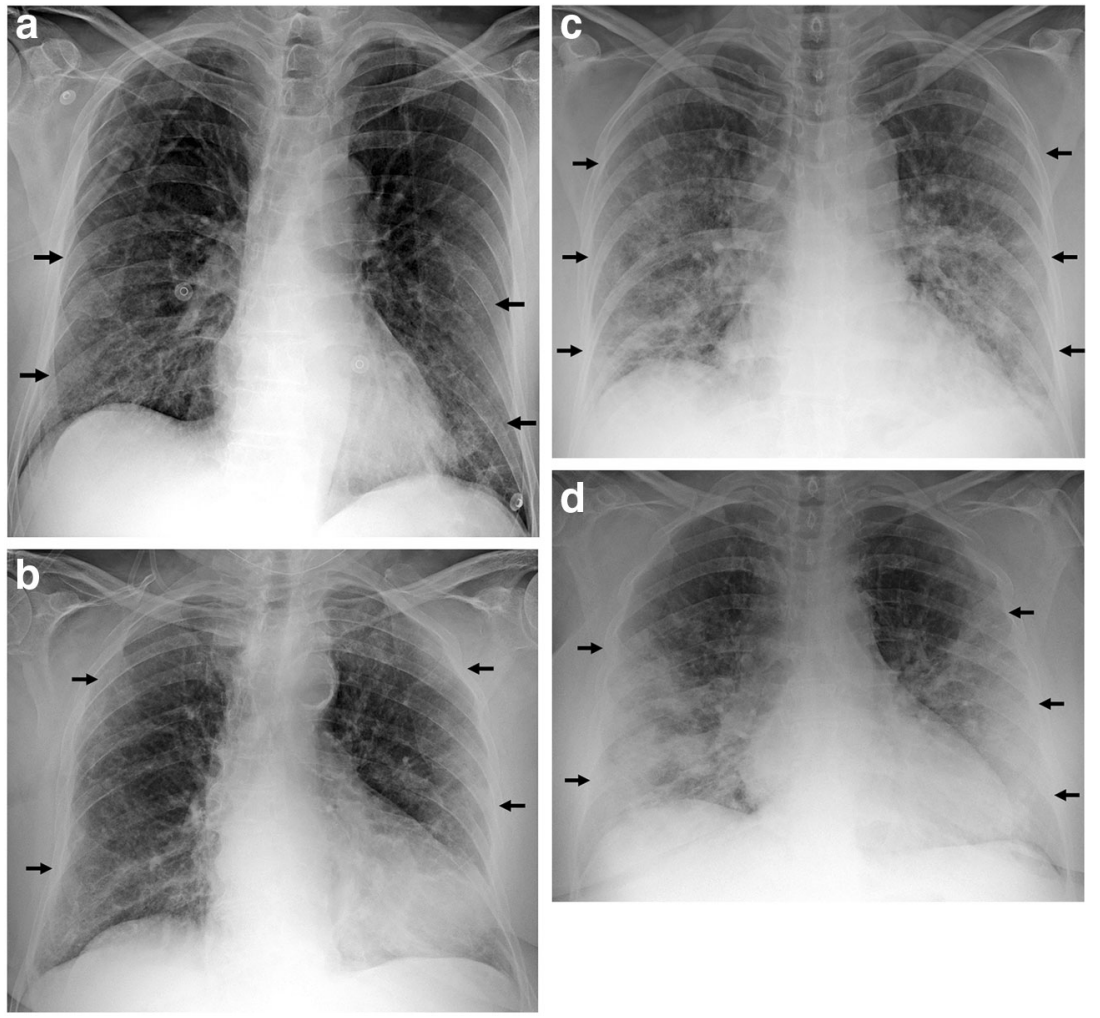

Fig. 2 a Group A, usual distribution. Chest radiography of a 78-year-old man, acquired within the first 2 days since the onset of symptoms, showing bilateral reticular alteration in the middle and lower fields (arrows). b Group B, usual distribution. Chest radiography of a 79-year-old woman, acquired within 3-5 days since the onset of symptoms, showing bilateral peripheral ground-glass opacities (arrows) and faint reticular alteration in the right lower field. $\mathbf{c}$ Group C, usual distribution. Chest radiography of a

findings of COVID-19 pneumonia and reporting an overall rate of negative baseline CXR examinations of $31 \%$. Similarly, in our experience, the overall rate of normal CXR

60-year-old woman, acquired within 6-9 days since the onset of symptoms, showing bilateral and symmetrical mixed patterns with groundglass opacification, patchy consolidations (arrows); reticular alteration in the right lower and middle fields. d Group D, usual distribution. Chest radiography of a 61-year-old woman, acquired after 10 days since the onset of symptoms, showing bilateral and symmetrical extensive consolidations (arrows)

findings in patients with positive RT-PCR was $25 \%$, versus an overall rate of normal chest $\mathrm{CT}$ of $0-22 \%$ [23-25] reported in the literature.

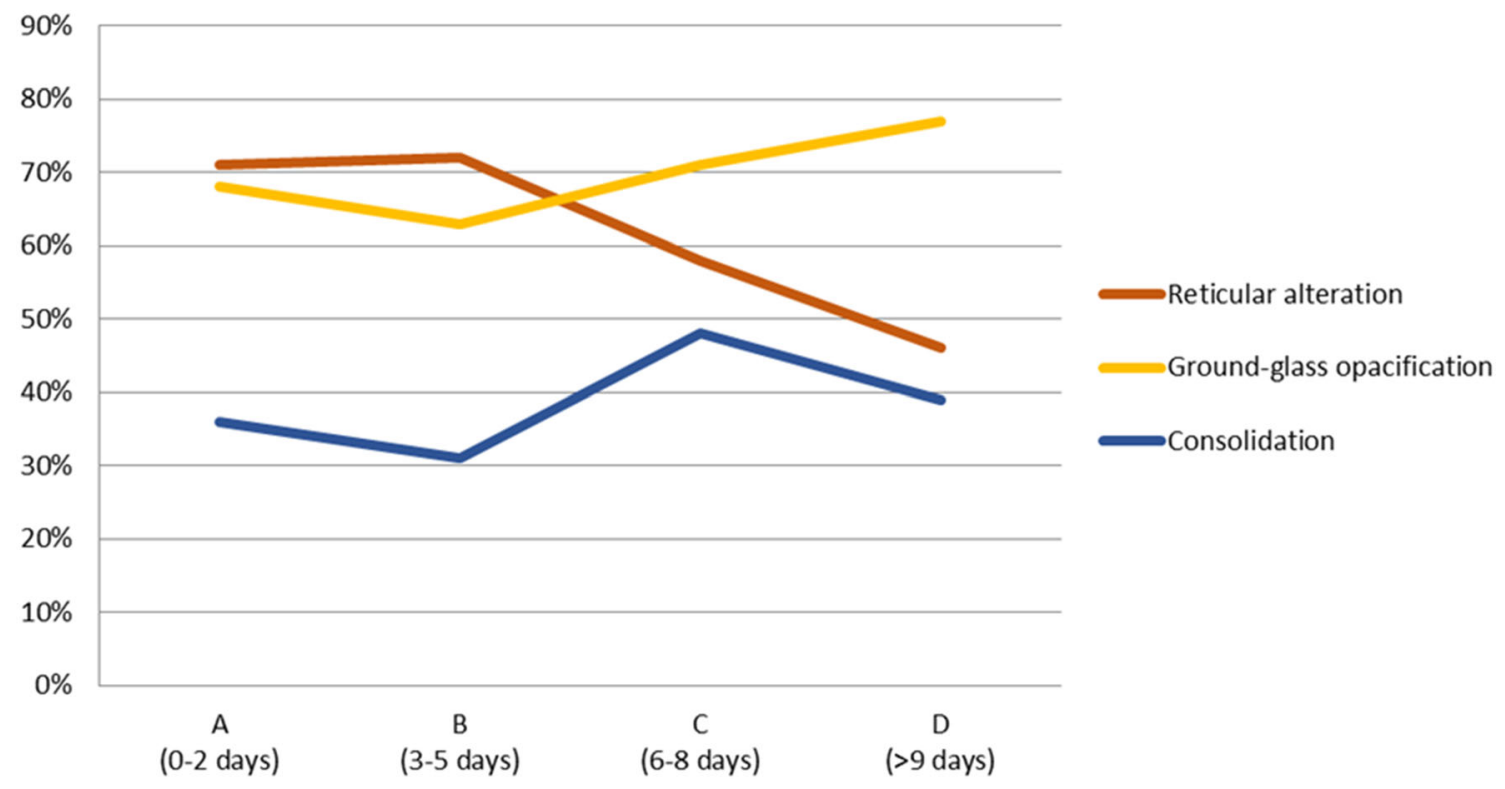

Fig. 3 The trend of the radiographic alterations in the different groups 
Fig. 4 The horizontal distribution (orange) and the craniocaudal distribution (green) of the alterations in the different groups
Horizontal distribution

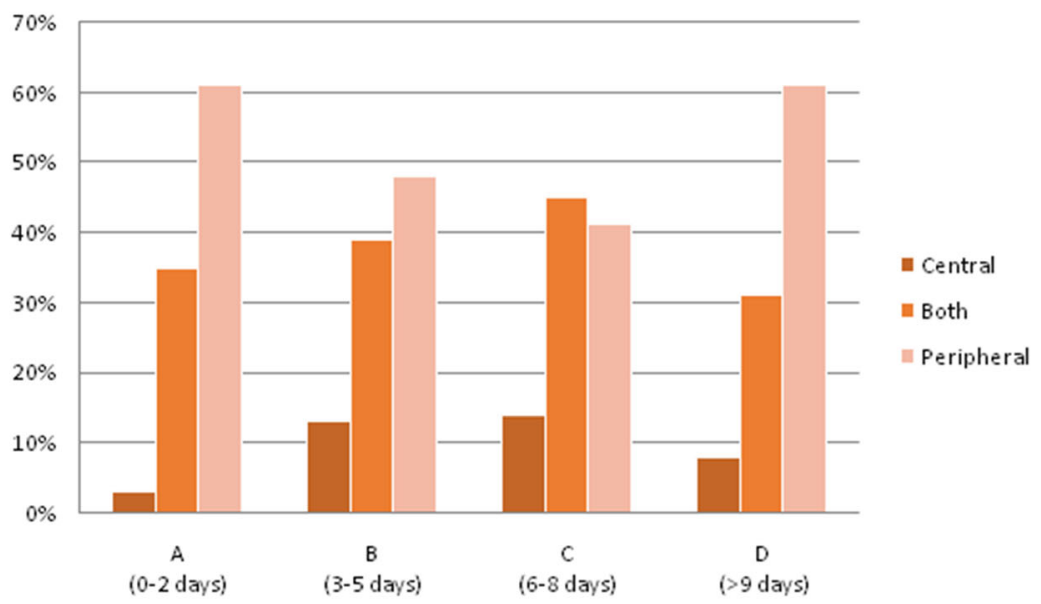

Cranio-caudal distribution

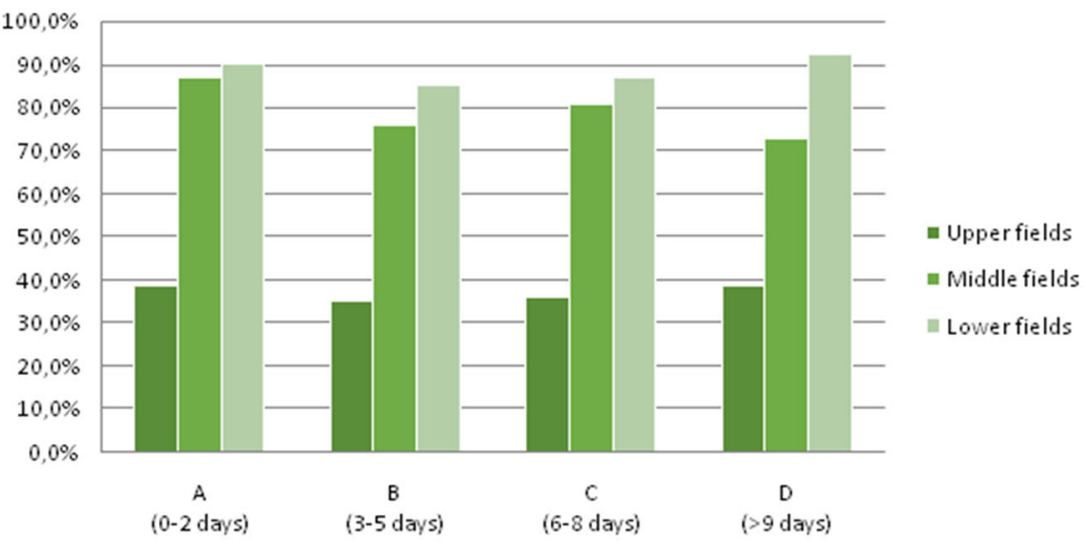

Bernheim et al [23] reported the percentage of normal chest $\mathrm{CT}$ in patients clustered on the base of the timing of symptom onset and observed a rapid decrease from $56 \%$ at $0-2$ days to $9 \%$ at 3-5 days and $4 \%$ at 6-12 days; initial RT-PCR was negative in $12 \%$ of the patients included in the study. Clustering our patients on the base of the timing of symptom onset, the rate of normal CXR progressively decreased from $36.7 \%$ (0-2 days after the onset of symptoms) to $28 \%$ (35 days), $18.8 \%$ (6-9 days), and $16.1 \%$ ( $>9$ days).

Although the two cohorts are not directly comparable due to different selection criteria and different diagnostic methods, we observed a decreasing trend in the rate of negative CXR through the different time intervals.

In our radiographic series, the most frequent alterations were GGO and reticular pattern, alone or in combination with other alterations, resembling the radiographic appearance described in other coronavirus-related pneumonias [26-28]. Furthermore, the distribution of the lesions in the middle fields and that of the relative sparing of the superior fields are similar to the pattern of distribution described in H1N1 influenza pneumonia [29]. In the first 5 days from the onset of the symptoms, reticular alteration was slightly more frequent than GGO, while after this period, GGO came to be predominant. Consolidation was constantly less frequent than the other two alterations, especially in the early phase of the disease.

Our data suggest that early alterations are predominantly reticular, intermediate alterations are predominantly GGO with a period of overlap between these two, whereas consolidations increase in the late phase. As known, the interobserver reliability of CXR is fair to good for the detection of pulmonary infiltrates, while this is poor for determining the pattern of alteration [30]. In line with this observation, in our study when disagreement was present between the two observers, it concerned in most cases the type of radiographic alteration, rather than the detection of alterations or their location.

Considering the distribution of the lesions on both the lungs and on the axial and craniocaudal plane, our results are in line with the data reported in CXR studies and in CT studies in the literature $[3,9,16,25]$.

In accordance with the observations of Wong et al [9], in the majority of our patients, the alterations were bilateral, and 
in patients with unilateral lesions, no predominance was observed between left and right.

The exclusive peripheral involvement and the combination of peripheral and central distributions were significantly more frequent than exclusive central distribution, without significant modifications of their proportions in the different time intervals.

Several studies reported a predominant, although not significant, localization of the lesions in the lower lobes [15, 23]; our results confirm this observation and measured a significantly higher frequency of involvement of the lower fields compared to the middle fields, and of the lower and middle fields compared to the upper fields.

Our patients had a higher mean age than those reported in the literature, reflecting demographic differences between China and the Western countries.

The purpose of this study was to analyze and describe the type, frequency, and distribution of CXR findings in COVID-19 pneumonia. Therefore, the main limitation of the study is the lack of data about the specificity of the CXR findings in COVID-19 towards its main differential diagnoses (other viral pneumonia, interstitial lung disease, cardiogenic pulmonary edema, acute lung injury), and the lack of correlation between CXR and CT findings. The description of the radiographic changes during the course of time was beyond the purpose of our study. Our study focused on the CXR at the admission, and the subsequent examinations of each patient were not considered; hence, the radiographic differences between the groups do not reflect the course of the radiographic changes over time, but rather the different radiographic presentations at different time intervals since the onset of symptoms. Another limitation of the present study is due to the lower quality of bedside radiographs compared to the PA radiographs. On the other hand, the advantage of portable bedside CXR consists of fewer requirement for decontamination of equipment, rooms, and hallways and, eventually, a lower risk of infection for other patients and healthcare workers.

In conclusion, the most frequent CXR lesions were reticular alteration and GGO, the first prevailing in the early phase, the following prevailing in the late phase. Consolidation was less frequent but, in agreement with other study observations, showed an increasing trend over time [16, 23].

Our observations are consistent with previous CXR and CT studies about the bilateral, peripheral, middle, and lower field predominant patterns of distribution of the lesions $[9,16,23$, 25]. In a pandemic scenario, with a high number of inpatients and a growing number of suspected cases, CXR should be considered as a feasible and easy-to-use method to assess lung involvement. Our results confirm the most recent recommendations, to employ CXR as a first-line imaging modality in the diagnostic workflow of patients with suspected COVID-19 pneumonia.
Funding information The authors state that this work has not received any funding.

\section{Compliance with ethical standards}

Guarantor The scientific guarantor of this publication is Prof. Lorenzo Preda.

Conflict of interest $\mathrm{CB}$ is a consultant for Bracco Imaging Italia and Doc. Congress. The other authors have nothing to disclose.

Statistics and biometry One of the authors has significant statistical expertise.

Informed consent Written informed consent was obtained from all subjects (patients) in this study.

Ethical approval Institutional review board approval was obtained.

\author{
Methodology \\ - retrospective \\ - observational \\ - performed at one institution
}

\section{References}

1. World Health Organization (2020) WHO Director-General's opening remarks at the media briefing on COVID-19. World Health Organization, Geneva Available via https://www.who.int/dg/ speeches/detail/who-director-general-s-opening-remarks-at-themedia-briefing-on-covid-19\%2D\%2D-11-march-2020. Accessed 26 Mar 2020

2. Huang C, Wang Y, Li X et al (2020) Clinical features of patients infected with 2019 novel coronavirus in Wuhan, China. Lancet 395:497-506

3. Shi H, Han X, Jiang N et al (2020) Radiological findings from 81 patients with COVID-19 pneumonia in Wuhan, China: a descriptive study. Lancet Infect Dis 20:425-434

4. World Health Organization (2020) Coronavirus disease (COVID19) technical guidance: laboratory testing for 2019-nCoV in humans. World Health Organization, Geneva Available via https://www.who.int/emergencies/diseases/novel-coronavirus2019/technical-guidance/laboratory-guidance. Accessed 26 Mar 2020

5. Ng M-Y, Lee EY, Yang J et al (2020) Imaging profile of the COVID-19 infection: radiologic findings and literature review. Radiology: Cardiothoracic Imaging. https://doi.org/10.1148/ryct. 2020200034

6. Song F, Shi N, Shan F et al (2020) Emerging 2019 novel coronavirus (2019-nCoV) pneumonia. Radiology 295:210-217

7. Rubin GD, Ryerson CJ, Haramati LB et al (2020) The role of chest imaging in patient management during the COVID-19 pandemic: a multinational consensus statement from the Fleischner Society. Radiology. https://doi.org/10.1148/radiol.2020201365:201365

8. Società Italiana di Radiologia Medica e Interventistica (2020) Documento intersocietario SIRM di marzo 2020. Società Italiana di Radiologia Medica e Interventistica, Milano. Available via https://www.sirm.org/wp-content/uploads/2020/03/DI. Accessed 26 Mar 2020

9. Wong HYF, Lam HYS, Fong AH et al (2019) Frequency and distribution of chest radiographic findings in COVID-19 positive 
patients. Radiology. https://doi.org/10.1148/radiol.2020201160: 201160

10. Yoon SH, Lee KH, Kim JY et al (2020) Chest radiographic and CT findings of the 2019 novel coronavirus disease (COVID-19): analysis of nine patients treated in Korea. Korean J Radiol 21:494-500

11. Salehi S, Abedi A, Balakrishnan S, Gholamrezanezhad A (2020) Coronavirus disease 2019 (COVID-19): a systematic review of imaging findings in 919 patients. AJR Am J Roentgenol. https://doi. org/10.2214/AJR.20.23034:1-7

12. Soldati G, Smargiassi A, Inchingolo R et al (2020) Is there a role for lung ultrasound during the COVID-19 pandemic? J Ultrasound Med. https://doi.org/10.1002/jum.15284

13. Hansell DM, Bankier AA, MacMahon H, McLoud TC, Muller NL, Remy J (2008) Fleischner Society: glossary of terms for thoracic imaging. Radiology 246:697-722

14. Timmerman R, McGarry R, Yiannoutsos C et al (2006) Excessive toxicity when treating central tumors in a phase II study of stereotactic body radiation therapy for medically inoperable early-stage lung cancer. J Clin Oncol 24:4833-4839

15. Zu ZY, Jiang MD, Xu PP et al (2020) Coronavirus disease 2019 (COVID-19): a perspective from China. Radiology. https://doi.org/ 10.1148/radiol.2020200490:200490

16. Pan F, Ye T, Sun P et al (2020) Time course of lung changes on chest CT during recovery from 2019 novel coronavirus (COVID19) pneumonia. Radiology. https://doi.org/10.1148/radiol. 2020200370:200370

17. BMJ Best Practice (2020) Coronavirus disease 2019 (COVID-19) British Medical Journal, London Available via https://bestpractice. bmj.com/topics/en-gb/3000168/diagnosis-approach. Accessed 26 Mar 2020

18. ACR (2020) ACR recommendations for the use of chest radiography and computed tomography (CT) for suspected COVID-19 infection. American College of Radiology, Virginia Available via https://www.acr.org/Advocacy-and-Economics/ACR-PositionStatements/Recommendations-for-Chest-Radiography-and-CTfor-Suspected-COVID19-Infection. Accessed 26 Mar 2020

19. RCR (2020) RCR position on the role of CT in patients suspected with COVID-19 infection. Royal College of Radiologists. Available via https://www.rcr.ac.uk/college/coronavirus-covid-19what-rcr-doing/rcr-position-role-ct-patients-suspected-covid-19. Accessed 26 Mar 2020
20. Society of Thoracic Radiology (2020) STR/ASER COVID-19 position statement. Society of Thoracic Radiology, Illinois Available via https://thoracicrad.org. Accessed 26 Mar 2020

21. Royal Australian and New Zealand College of Radiologists (2020) COVID-19 essential role of clinical radiology services position statement. Royal Australian and New Zealand College of Radiologists, Sydney Available via https://www.ranzcr.com/ourwork/coronavirus\#advic. Accessed 26 Mar 2020

22. Canadian Society of Thoracic Radiology and the Canadian Association of Radiologists (2020) Canadian Society of Thoracic Radiology and the Canadian Association of Radiologists' statement on COVID-19. Canadian Society of Thoracic Radiology and the Canadian Association of Radiologists, Ottawa Available via https://carca/cstr. Accessed 26 Mar 2020

23. Bernheim A, Mei X, Huang M et al (2020) Chest CT findings in coronavirus disease-19 (COVID-19): relationship to duration of infection. Radiology. https://doi.org/10.1148/radiol.2020200463: 200463

24. Kanne JP (2020) Chest CT findings in 2019 novel coronavirus (2019-nCoV) infections from Wuhan, China: key points for the radiologist. Radiology 295:16-17

25. Chung M, Bernheim A, Mei X et al (2020) CT imaging features of 2019 novel coronavirus (2019-nCoV). Radiology 295:202-207

26. Muller NL, Ooi GC, Khong PL, Nicolaou S (2003) Severe acute respiratory syndrome: radiographic and CT findings. AJR Am J Roentgenol 181:3-8

27. Franquet $T$ (2011) Imaging of pulmonary viral pneumonia. Radiology 260:18-39

28. Koo HJ, Lim S, Choe J, Choi SH, Sung H, Do KH (2018) Radiographic and CT features of viral pneumonia. Radiographics 38:719-739

29. Aviram G, Bar-Shai A, Sosna J et al (2010) H1N1 influenza: initial chest radiographic findings in helping predict patient outcome. Radiology 255:252-259

30. Albaum MN, Hill LC, Murphy M et al (1996) Interobserver reliability of the chest radiograph in community-acquired pneumonia. PORT Investigators. Chest 110:343-350

Publisher's note Springer Nature remains neutral with regard to jurisdictional claims in published maps and institutional affiliations. 